\title{
Motion in the Restricted Three-Body Problem at the Nanoscale
}

\author{
Jagadish Singh ${ }^{1, a}$ and Tyokyaa K. Richard ${ }^{2, b^{*}}$
}

\author{
Department of Mathematics, Faculty of Physical Sciences, Ahmadu Bello University, Zaria, Nigeria. \\ Department of Mathematical Sciences, Faculty of Physical Sciences, \\ Federal University Dutsin-Ma, Katsina State, Nigeria.
}

ae-mail: jgds2004@yahoo.com, be-mail: rtkanshio6@gmail.com

\begin{abstract}
Keywords: Carbon atom, Nanoscale, $C_{60}$ fullerenes, Positions, Stability, Restricted Three-Body Problem.
\end{abstract}

\begin{abstract}
This paper studies the classical restricted three-body problem of a carbon atom in the vicinity of two carbon 60 fullerenes ( $C_{60}$ fullerenes) at the nanoscale. The total molecular energy between the two fullerenes is determined analytically by approximating the pairwise potential energies between the carbon atoms on the fullerenes by a continuous approach. Using software MATHEMATICA, we compute the positions of the stationary points and their stability for a carbon atom at the nanosacle and it is observed that for each set of values, there exists at least one complex root with the positive real part and hence in the Lyapunov sense, the stationary points are unstable. Since only attractive Van der Waals forces contribute to the orbiting behavior, no orbiting phenomenon can be observed for $r<10 A^{O}$, where the Van der Waals forces becomes repulsive. Although the $C_{60}$-nanotorus orbital is speculative in nature and also presents exciting possibilities, there are still many practical challenges that would need to be overcome before the $C_{60}$ - nanotorus orbital might be realized. However, the present theoretical study is a necessary precursor to any of such developments.
\end{abstract}

\section{Introduction}

Industrial carbons have gone through a rapid development during the last decade. Carbon disc-brakes, electrodes and materials for medical uses represent only a few limited selection of products resulting from well-aimed advancements. The discovery of the fullerenes and carbon with nanotubes, the carbon science has remarkably developed owing to their unique mechanical and electronic nature such as the flexibility, high strength, low weight and their thermal stability. The study of carbon nanotubes and $C_{60}$ fullerenes are of great interest in various scientific areas. One of such is the creation of high-frequency nanoscale oscillators or gigahertz oscillators for ultrafast optical filters and nanoantennae applications. While there are difficulties for micromechanical oscillators or resonators to reach a frequency in the gigahertz range, it is possible for nanomechanical systems.

Jones [1], in the new scientist, suggested that, the hollow all-carbon cage molecules were possible under the name of Daedalus. Two decades later, reference [2], when analyzing the resulting mass spectrometer, discovered the carbon 60 and 70 fullerenes $\left(C_{60}\right.$ and $C_{70}$ fullerenes).

Zheng et al. [3] observed how the sliding of the inner shell inside the outer shell of a multi-walled carbon nanotube can generate oscillatory frequencies up to several gigahertz in the molecular gigahertz oscillators. Reference [4] pointed out that, instead of using multi-walled carbon nanotubes in generating oscillatory frequencies up to several gigahertz, a fullerene $C_{60}$ oscillating inside a singlewalled carbon nanotube be used. Various nanoscale gigahertz oscillators have been studied, the oscillation of $C_{60}$ fullerene on a multi-walled carbnon nanotube is one of such. Cox et al. [5] in their molecular dynamics simulations of various nano-gigahertz oscillators deployed a mathematical model of fundamental mechanical principles and classical applied mathematical techniques to determine an acceptable condition and the suction energies of a $C_{60}$ fullerene entering a nanotube. The various two body problem namely; fullerene-fullerene and fullerene-carbon nanotube at the nanoscale were investigated by [6]. 
Chan et al. [7] in their study of stationary points of the circular planar restricted three-body problem for three $C_{60}$ fullerenes and a carbon atom and two $C_{60}$ fullerenes affirmed that the maximum angular frequency of the two and three fullerenes systems reached the terahertz range.

In the present study, we wish to extend the work of [7] to include the stability of the stationary points which have maximum velocity for the carbon atom and the two fullerenes system neglecting any thermal fluctions arising from the environment.

The paper is divided into 8 sections; in section 2, the equations of motion of the restricted three-body problem for the three $C_{60}$ fullerene systems are outlined. In section 3, the equations of three identical $C_{60}$ fullerenes are studied. The equations of motion of a carbon atom are considered in section 4 . The positions of a carbon atom and two fullerenes are examined in section 5. In section 6, the stability of motion for the aforementioned problem is discussed. The numerical applications are shown in section 7 while the discussion and conclusion are presented in section 8 .

\section{Equations of Motion}

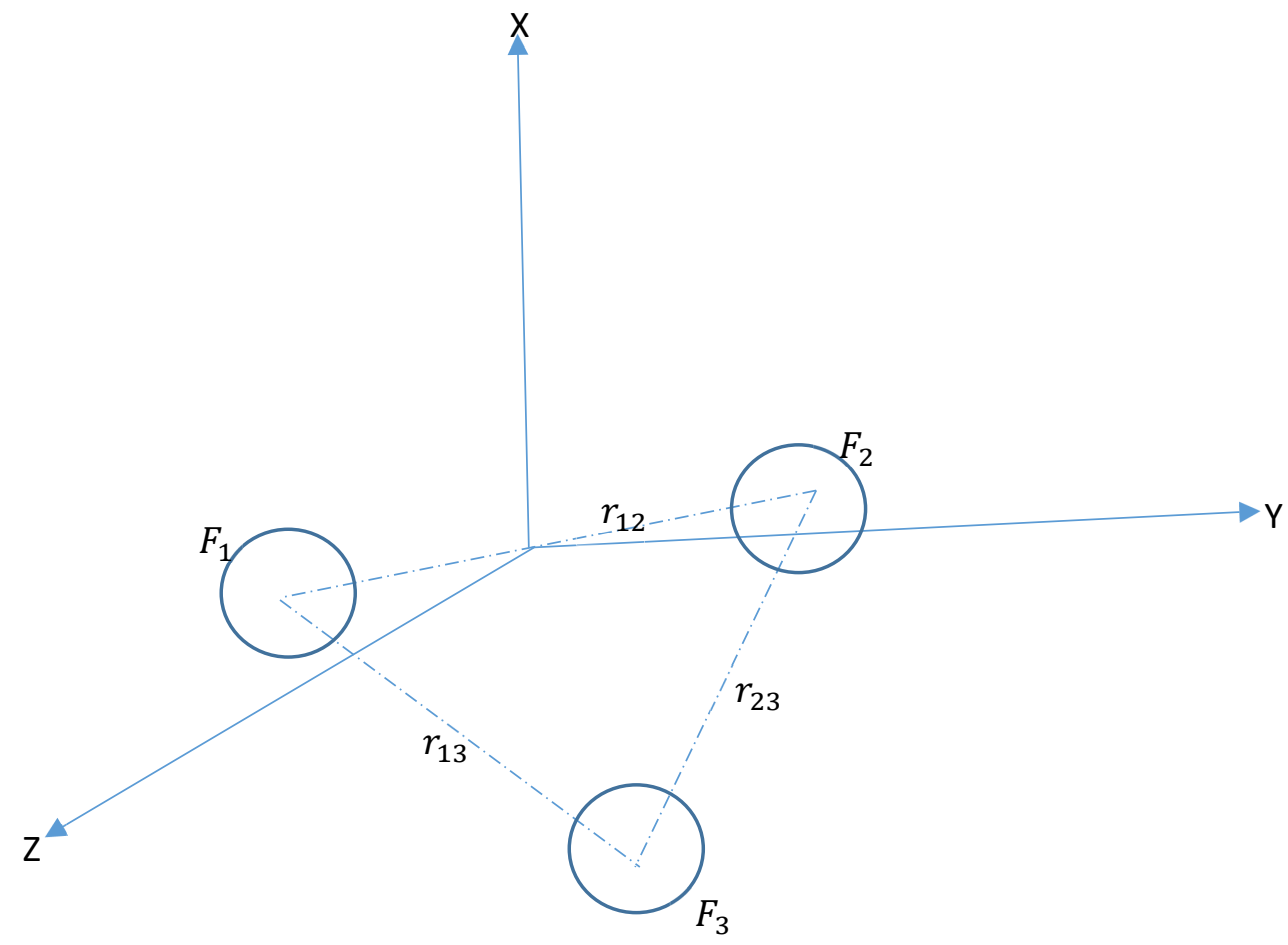

Fig. 1: Three-body problem at the nanoscale

Consider the three-body problem at the nanosacale with fullerene centers lying at the vertices of an equilateral triangle. Since we are considering the residual molecular interactions between molecules, it is pertinent to investigate the same problem at the nanoscale. We for this adopt the Lennard-Jones potential [8] given as thus;

$$
\psi(\tau)=4 \varepsilon\left[\left(\frac{\alpha}{\tau}\right)^{12}-\left(\frac{\alpha}{\tau}\right)^{6}\right]=-\frac{A}{\tau^{6}}+\frac{B}{\tau^{12}}
$$

where $\tau, \varepsilon, \alpha, A$ and $B$ are respectively the distance between two arbitrary carbom atoms, the potential well depth of the two carbon atoms, the parameter that is determined by the equilibrium distance, the attractive constant and the repulsive constant. With the assumption that the carbon atoms are distributed evenly over the surfaces of the molecules, according to [5], the pairwise molecular energy between two fullerenes given as $\sum_{k} \sum_{j} \psi\left(\tau_{k j}\right)$ can be approximated by continuous approach as

$$
\psi(r)=\mathrm{n}_{f}^{2} \int_{s_{1}} \int_{s_{2}}\left(-\frac{A}{\tau^{6}}+\frac{B}{\tau^{12}}\right) d s_{2} d s_{1}
$$


where $n_{f}$ and $\tau$ are the atomic number density and the distance between the centres of two fullerenes, respectively. The surface area elements of the two fullerenes are denoted by $d s_{1}$ and $d s_{2}$.

Now,

$$
\psi(r)=-Q_{6}(r)+Q_{12}(r)
$$

where $Q_{n}$ is defined by

$$
Q_{n}(r)=\frac{4 \pi^{2} a^{2} C_{n} \mathrm{n}_{f}^{2}}{r(n-2)(n-3)}\left(\frac{1}{(2 a+r)^{n-3}}-\frac{1}{(2 a-r)^{n-3}}-\frac{2}{r^{n-3}}\right)
$$

where $a, C_{6}$ and $C_{12}$ are the radius of the $C_{60}$ fullerene, $A$ and $B$ respectively.

Now, the mutual force $F(r)$ between two fullerenes can be given as;

$$
F(r)=-\frac{d \psi(r)}{d r}
$$

By Newton's second law of motion, the force acting on a fullerene by the other two fullerenes is given as

$$
m_{k} \ddot{\overrightarrow{r_{k}}}=\sum_{j \neq k=1}^{3} F\left(r_{k j}\right) \frac{\overrightarrow{r_{j}}-\overrightarrow{r_{k}}}{r_{k j}}
$$

where $m_{k}, r_{k}$, and $r_{k j}$ are respectively the total mass of the $k$-fullerene, the position vector of the $k$-fullerene and the relative displacement of any two fullerenes $k$ and $j$.

We let $\vec{r}=\overrightarrow{r_{2}}-\overrightarrow{r_{1}}, \vec{R}$ to be the center of mass of $m_{1}$ and $m_{2}$ allocated at the origin of the coordinate system and $\vec{\tau}=\overrightarrow{r_{3}}-\vec{R}=M \mu^{-1} \overrightarrow{r_{3}}$ where $\mu=m_{1}+m_{2}$. With these assumptions, we have;

$$
\begin{aligned}
& \overrightarrow{r_{2}}-\overrightarrow{r_{1}}=\vec{r} \\
& \overrightarrow{r_{3}}-\overrightarrow{r_{1}}=\vec{\tau}+m_{2} \mu^{-1} \vec{r} \\
& \overrightarrow{r_{3}}-\overrightarrow{r_{2}}=\vec{\tau}-m_{1} \mu^{-1} \vec{r}
\end{aligned}
$$

Therefore, the equations of motion in terms of the Jacobian coordinates $\vec{r}$ and $\vec{\tau}$ are then given by

$$
\begin{aligned}
& \ddot{\vec{r}}=\frac{-\mu}{m_{1} m_{2}} \frac{F\left(r_{12}\right) \vec{r}}{r_{12}}+\frac{1}{m_{2}} \frac{F\left(r_{23}\right)}{r_{23}}\left(\vec{\tau}-m_{1} \mu^{-1} \vec{r}\right)-\frac{1}{m_{1}} \frac{F\left(r_{13}\right)}{r_{13}}\left(\vec{\tau}+m_{2} \mu^{-1} \vec{r}\right) \\
& \ddot{\vec{\tau}}=\frac{-M \mu^{-1}}{m_{3}}\left(\frac{F\left(r_{13}\right)}{r_{13}}\left(\vec{\tau}+m_{2} \mu^{-1} \vec{r}\right)+\frac{F\left(r_{23}\right)}{r_{23}}\left(\vec{\tau}-m_{1} \mu^{-1} \vec{r}\right)\right)
\end{aligned}
$$

\section{Three Identical $\boldsymbol{C}_{60}$ Fullerenes}

We assume that the three $C_{60}$ fullerenes are moving uniformly in circular orbits with the same angular velocities in the same plane. We denote $\left(x_{i}, y_{i}, 0\right)$ as the coordinates of the $i$-fullerenes. Then from Eq. (6), we have

$$
\begin{gathered}
\ddot{x_{k}}=m_{k}^{-1} \sum_{j \neq k} \frac{F\left(r_{j k}\right)}{r_{j k}}\left(x_{j}-x_{k}\right) \\
\ddot{y_{k}}=m_{k}^{-1} \sum_{j \neq k} \frac{F\left(r_{j k}\right)}{r_{j k}}\left(y_{j}-y_{k}\right)
\end{gathered}
$$

Considering the angular velocity $\omega$, the rotating coordinate system is given as

$$
\begin{gathered}
x_{k}=\xi_{k} \cos \omega t-\eta_{k} \sin \omega t \\
y_{k}=\xi_{k} \sin \omega t+\eta_{k} \cos \omega t
\end{gathered}
$$

From which we obtain,

$$
\begin{aligned}
& \ddot{x}_{k}=\ddot{\xi}_{k}-2 \omega \dot{\eta_{k}}-\omega^{2} \xi_{k} \\
& \ddot{y}_{k}=\ddot{\eta_{k}}+2 \omega \dot{\xi_{k}}-\omega^{2} \eta_{k}
\end{aligned}
$$

Making use of Eq. (10) and Eq. (11) in Eq. (9), we have 


$$
\begin{gathered}
\ddot{\xi}_{k}-2 \omega \dot{\eta_{k}}-\omega^{2} \xi_{k}=m_{k}^{-1} \sum_{j \neq k} \frac{F\left(r_{j k}\right)}{r_{j k}}\left(\xi_{j}-\xi_{k}\right) \\
\ddot{\eta_{k}}+2 \omega \dot{\xi_{k}}-\omega^{2} \eta_{k}=m_{k}^{-1} \sum_{j \neq k} \frac{F\left(r_{j k}\right)}{r_{j k}}\left(\eta_{j}-\eta_{k}\right)
\end{gathered}
$$

With the assumption that the three fullerenes are identical and allocated at the vertices of an equilateral triangle, i.e; $m_{1}=m_{2}=m_{3}=m, \mu=m_{1}+m_{2}=2 m, r_{12}=r_{13}=r_{23}=r$ and on assuming $F\left(r_{12}\right)=F\left(r_{13}\right)=F\left(r_{23}\right)=F(r)$, the first equation of (8) yields

$$
\ddot{\vec{r}}=\frac{-3 F(r) \vec{r}}{m r}
$$

This represents a simple harmonic motion with the angular velocity $\omega=\sqrt{\frac{3 F(r)}{m r}}$.

\section{Equations of Motion of a Carbon Atom.}

We now examine the motion of a carbon atom under the influences of two $C_{60}$ identical fullerenes. We suppose that the molecular interaction of the carbon is negligible so that it has no effect on the dynamics of the two $C_{60}$ fullerenes which are moving with angular velocity as in circular orbits of radius $R$ in a plane. Then Eq. (8) becomes

$$
\begin{aligned}
& \ddot{\vec{r}}=\frac{-2}{m} \frac{F\left(r_{12}\right) \vec{r}}{r_{12}} \\
& \ddot{\vec{\tau}}=\frac{-1}{m_{c}}\left(\frac{F\left(\tau_{1}\right)}{\tau_{1}}\left(\vec{\tau}+\frac{\vec{r}}{2}\right)+\frac{F\left(\tau_{2}\right)}{\tau_{2}}\left(\vec{\tau}-\frac{\vec{r}}{2}\right)\right)
\end{aligned}
$$

where $m, m_{c}, \tau_{1}$ and $\tau_{2}$ are respectively the mass of $C_{60}$ molecule, the mass of the carbon atom, the distances between the atom and the first and second fullerenes. The first equation of Eq. (14) represents two-body problem and describes the motion of the two $C_{60}$ identical fullerenes. It also shows a simple harmonic motion with

$$
\omega=\sqrt{\frac{2 F(r)}{m r}} \text { for a fixed } r \text {. }
$$

The second equation of Eq. (14) provides the motion of a carbon atom. We take a Cartesian coordinate system $(0, \xi, \eta)$ rotating with angular velocity $\omega$ and the origin at the centre of mass of the two fullerenes. The positions of the two fullerenes are $\left(-\frac{R}{2}, 0\right)$ and $\left(\frac{R}{2}, 0\right)$ where $R$ is the distance between them. The equations of motion of the carbon atom under the aforesaid circular planar assumptions can be obtained from Eq. (12) as

$$
\begin{aligned}
& \ddot{\xi}-2 \omega \dot{\eta}-\omega^{2} \xi=\frac{1}{m_{c}}\left(\frac{F\left(\tau_{1}\right)}{\tau_{1}}\left(-\frac{R}{2}-\xi\right)+\frac{F\left(\tau_{2}\right)}{\tau_{2}}\left(\frac{R}{2}-\xi\right)\right) \\
& \ddot{\eta}+2 \omega \dot{\xi}-\omega^{2} \eta=\frac{1}{m_{c}}\left(\frac{F\left(\tau_{1}\right)}{\tau_{1}}(0-\eta)+\frac{F\left(\tau_{2}\right)}{\tau_{2}}(0-\eta)\right)
\end{aligned}
$$

Where $(\xi, \eta)$ are coordinates of the carbon atom of mass $m_{c}$ and $\tau_{1}=\sqrt{\left(\xi+\frac{R}{2}\right)^{2}+\eta^{2}}, \tau_{2}=$ $\sqrt{\left(\xi-\frac{R}{2}\right)^{2}+\eta^{2}}$ are its distances from the two fullerenes.

That is

$$
\begin{aligned}
& \ddot{\xi}-2 \omega \dot{\eta}=\frac{1}{2 m_{c}} \frac{\partial Q}{\partial \xi} \\
& \ddot{\eta}+2 \omega \dot{\xi}=\frac{1}{2 m_{c}} \frac{\partial Q}{\partial \eta}
\end{aligned}
$$

where $Q=Q_{1}+Q_{2}$ and $Q_{1}=\psi(\tau)+m_{c} \omega^{2} \xi^{2}, Q_{2}=\psi(\tau)+m_{c} \omega^{2} \eta^{2}$. 


\section{Positions of Collinear Equilibrium Points of the Carbon Atom}

To determine the position of the carbon atom with respect to the rotating frame under the influence of the two $C_{60}$ fullerenes, we set $\dot{\xi}=\dot{\eta}=0=\ddot{\xi}=\ddot{\eta}$ in Eq. (15) and thus solve equations $\frac{\partial Q}{\partial \xi}=$ $0=\frac{\partial Q}{\partial \eta}$ for $\xi$ and $\eta$. Given that;

$$
Q=\frac{-A}{\left(\left(\xi+\frac{R}{2}\right)^{2}+\eta^{2}\right)^{3}}+\frac{B}{\left(\left(\xi+\frac{R}{2}\right)^{2}+\eta^{2}\right)^{6}}-\frac{A}{\left(\left(\xi-\frac{R}{2}\right)^{2}+\eta^{2}\right)^{3}}+\frac{B}{\left(\left(\xi-\frac{R}{2}\right)^{2}+\eta^{2}\right)^{6}}+m_{C} \omega^{2}\left(\xi^{2}+\eta^{2}\right)
$$

Now,

$$
\begin{aligned}
& \frac{\partial Q}{\partial \xi}=\frac{-12 B\left(\xi-\frac{R}{2}\right)}{\left(\eta^{2}+\left(\xi-\frac{R}{2}\right)^{2}\right)^{7}}+\frac{6 A\left(\xi-\frac{R}{2}\right)}{\left(\eta^{2}+\left(\xi-\frac{R}{2}\right)^{2}\right)}-\frac{12 B\left(\xi+\frac{R}{2}\right)}{\left(\eta^{2}+\left(\xi+\frac{R}{2}\right)^{2}\right)^{7}}+\frac{6 A\left(\xi+\frac{R}{2}\right)}{\left(\eta^{2}+\left(\xi+\frac{R}{2}\right)^{2}\right)^{4}}+2 \xi m_{c} \omega^{2} \\
& \frac{\partial Q}{\partial \eta}=\frac{-12 B \eta}{\left(\eta^{2}+\left(\xi-\frac{R}{2}\right)^{2}\right)^{7}}+\frac{6 A \eta}{\left(\eta^{2}+\left(\xi-\frac{R}{2}\right)^{2}\right)^{4}}-\frac{12 B \eta}{\left(\eta^{2}+\left(\xi+\frac{R}{2}\right)^{2}\right)^{7}}+\frac{6 A \eta}{\left(\eta^{2}+\left(\xi+\frac{R}{2}\right)^{2}\right)^{4}}+2 \eta m_{c} \omega^{2}
\end{aligned}
$$

At stationary points $\frac{\partial Q}{\partial \xi}=\frac{\partial Q}{\partial \eta}=0$ that is

$$
\begin{aligned}
& \frac{-12 B\left(\xi-\frac{R}{2}\right)}{\left(\eta^{2}+\left(\xi-\frac{R}{2}\right)^{2}\right)^{7}}+\frac{6 A\left(\xi-\frac{R}{2}\right)}{\left(\eta^{2}+\left(\xi-\frac{R}{2}\right)^{2}\right)^{4}}-\frac{12 B\left(\xi+\frac{R}{2}\right)}{\left(\eta^{2}+\left(\xi+\frac{R}{2}\right)^{2}\right)^{7}}+\frac{6 A\left(\xi+\frac{R}{2}\right)}{\left(\eta^{2}+\left(\xi+\frac{R}{2}\right)^{2}\right)^{4}}+2 \xi m_{C} \omega^{2}=0 \\
& \frac{-12 B \eta}{\left(\eta^{2}+\left(\xi-\frac{R}{2}\right)^{2}\right)^{7}}+\frac{6 A \eta}{\left(\eta^{2}+\left(\xi-\frac{R}{2}\right)^{2}\right)^{4}}-\frac{12 B \eta}{\left(\eta^{2}+\left(\xi+\frac{R}{2}\right)^{2}\right)^{7}}+\frac{6 A \eta}{\left(\eta^{2}+\left(\xi+\frac{R}{2}\right)^{2}\right)^{4}}+2 \eta m_{c} \omega^{2}=0
\end{aligned}
$$

With $A=17.4, B=29 \times 10^{3}, R=10.5, m_{c}=1.993 \times 10^{-26}, \omega=6 \times 10^{11} \quad$ we have equations (21) and (22) reduce to

$$
\begin{aligned}
& 0.0143496 \xi-\frac{348000(-5.25+\xi)}{\left(\eta^{2}+(-5.25+\xi)^{2}\right)^{7}}+\frac{1044(-5.25+\xi)}{\left(\eta^{2}+(-5.25+\xi)^{2}\right)^{4}}-\frac{348000(5.25+\xi)}{\left(\eta^{2}+(5.25+\xi)^{2}\right)^{7}}+\frac{1044(5.25+\xi)}{\left(\eta^{2}+(5.25+\xi)^{2}\right)^{4}}=0 \\
& 0.0143496 \eta-\frac{348000 \eta}{\left(\eta^{2}+(-5.25+\xi)^{2}\right)^{7}}+\frac{1044 \eta}{\left(\eta^{2}+(-5.25+\xi)^{2}\right)^{4}}-\frac{348000 \eta}{\left(\eta^{2}+(5.25+\xi)^{2}\right)^{7}}+\frac{1044 \eta}{\left(\eta^{2}+(5.25+\xi)^{2}\right)^{4}}=0
\end{aligned}
$$

Using the software MATHEMATICA, the positions of stationary points are shown in table 2 and Fig. 2 \& Fig. 3.

Table 1: Numerical data

\begin{tabular}{llcc}
\hline \hline S/NO. & CONSTANT NAME & SYMBOL & VALUE \\
\hline 1. & Radius of $C_{60}$ & $a$ & $3.55_{A}^{o}$ \\
2. & Carbon-Carbon bond length & $\alpha$ & $1.421_{A}^{o}$ \\
3. & Mean surface density of $C_{60}$ & $n_{f}$ & $0.3789_{A^{-2}}^{o}$ \\
4. & Mass of a single carbon atom & $m_{c}$ & $1.993 \times 10^{-26} \mathrm{~kg}$ \\
5. & Mass of a single $C_{60}$ fullerene & $m$ & $1.196 \times 10^{-24} \mathrm{~kg}$ \\
6. & Attractive constant & $A$ & $17.4 \mathrm{eV} \times{ }_{A^{6}}^{\circ}$ \\
7. & Repulsive constant & $B$ & $29 \times 10^{3} \mathrm{eV} \times 10^{12}$ \\
\hline \hline
\end{tabular}

Table 2: Positions of collinear equilibrium points of the carbon atom

\begin{tabular}{ccccccc}
\hline \hline $\boldsymbol{A}$ & $\boldsymbol{B}$ & $\boldsymbol{R}$ & $\boldsymbol{m}_{\boldsymbol{c}}$ & $\boldsymbol{\omega}$ & $\boldsymbol{\xi}$ & $\pm \boldsymbol{\eta}$ \\
\hline 17.4 & $29 \times 10^{3}$ & 10.5 & $1.993 \times 10^{-26}$ & $6 \times 10^{11}$ & 0 & 0 \\
& & & & -8.32285 & 0 \\
& & & & 8.32285 & 0
\end{tabular}




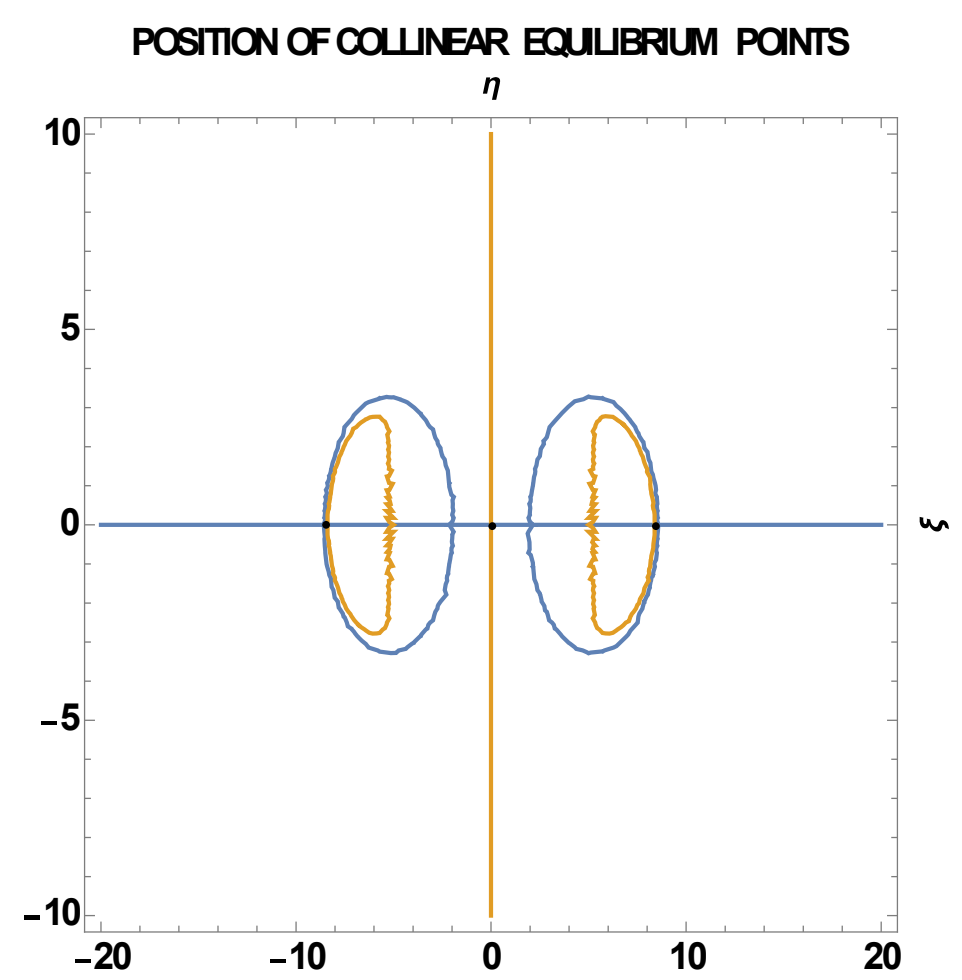

Fig. 2: Positions of collinear equilibrium points of the carbon atom

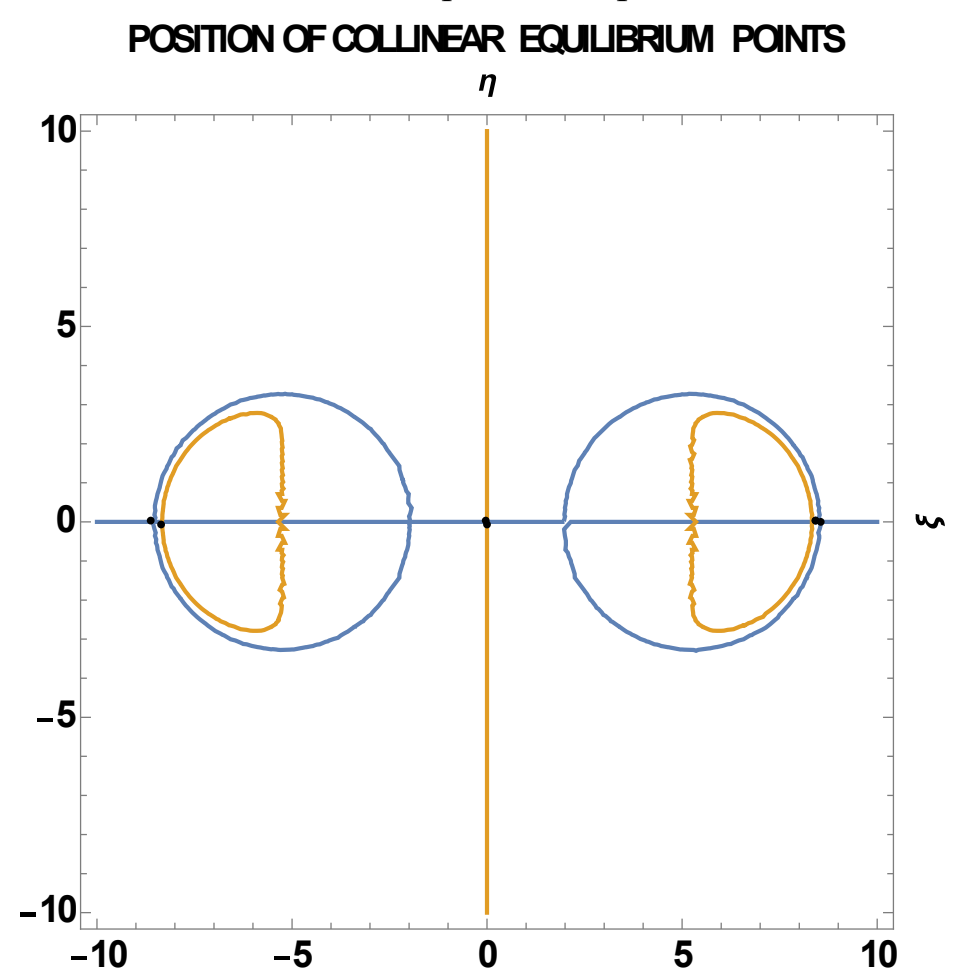

Fig. 3: Positions of collinear equilibrium points of the carbon atom

\section{Stability of Collinear Equilibrium Points of the Carbon Atom}

To examine the stability of the motion of a carbon atom in the vicinity of two $C_{60}$ fullerenes, we establish the characteristic equation of the system under review. To obtain the variational equations, we denote the position of a stationary point of a carbon atom by $\left(\xi_{o}, \eta_{o}\right)$ and then assume the small displacements in $\left(\xi_{o}, \eta_{o}\right)$ to be $(\sigma, \beta)$, and writing $\Omega$ for $Q$ in Eq. (15). Then we have $\xi=\xi_{o}+\sigma$ and $\eta=\eta_{o}+\beta$

Taking the derivatives we obtain 


$$
\begin{gathered}
\dot{\xi}=\dot{\sigma}, \ddot{\xi}=\ddot{\sigma} \\
\dot{\eta}=\dot{\beta}, \ddot{\eta}=\ddot{\beta}
\end{gathered}
$$

Making use of Eq. (25) into Eq. (15) yields the variational equations of motion:

$\ddot{\sigma}-2 \omega \dot{\beta}=\frac{1}{2 m_{c}}\left[\left(\Omega_{\xi \xi}^{0}\right) \sigma+\left(\Omega_{\xi \eta}^{0}\right) \beta\right]$

$\ddot{\beta}+2 \omega \dot{\sigma}=\frac{1}{2 m_{c}}\left[\left(\Omega_{\eta \xi}^{0}\right) \sigma+\left(\Omega_{\eta \eta}^{0}\right) \beta\right]$

The superscript $\mathrm{o}$ indicates that the partial derivatives are evaluated at the point under consideration Let

$$
\begin{aligned}
& \sigma=k_{1} e^{\lambda t}, \beta=k_{2} e^{\lambda t} \\
& \dot{\sigma}=k_{1} \lambda e^{\lambda t}, \dot{\beta}=k_{2} \lambda e^{\lambda t} \\
& \ddot{\sigma}=k_{1} \lambda^{2} e^{\lambda t}, \ddot{\beta}=k_{2} \lambda^{2} e^{\lambda t}
\end{aligned}
$$

Substituting Eq. (27) into Eq. (26) we have

$$
\begin{gathered}
\left(2 m_{c} \lambda^{2}-\Omega_{\xi \xi}^{0}\right) k_{1}+\left(-4 \omega m_{c} \lambda-\Omega_{\xi \eta}^{0}\right) k_{2}=0 \\
\left(4 \omega m_{c} \lambda-\Omega_{\eta \xi}^{0}\right) k_{1}+\left(2 m_{c} \lambda^{2}-\Omega_{\eta \eta}^{0}\right) k_{2}=0
\end{gathered}
$$

Which have a nontrivial solution if

$\left|\begin{array}{cc}2 m_{c} \lambda^{2}-\Omega_{\xi \xi}^{0} & -4 \omega m_{c} \lambda-\Omega_{\xi \eta}^{0} \\ 4 \omega m_{c} \lambda-\Omega_{\eta \xi}^{0} & 2 m_{c} \lambda^{2}-\Omega_{\eta \eta}^{0}\end{array}\right|=0$

Expanding the determinant, we have

$$
\lambda^{4}-\left[\frac{1}{2 m_{c}}\left(\Omega_{\xi \xi}^{0}+\Omega_{\eta \eta}^{0}-8 \omega^{2} m_{c}\right)\right] \lambda^{2}+\left[\frac{\omega}{m_{c}}\left(\Omega_{\xi \eta}^{0}-\Omega_{\eta \xi}^{0}\right)\right] \lambda+\left[\frac{1}{4 m_{c}^{2}}\left(\Omega_{\xi \xi}^{0} \Omega_{\eta \eta}^{0}-\Omega_{\xi \eta}^{0} \Omega_{\eta \xi}^{0}\right)\right]=0
$$

Eq. (29) is the characteristic equation for the stated problem.

From Eq. (19) and Eq. (20) the first order partial derivatives of $\Omega$ (on changing $Q$ into $\Omega$ ) are;

$$
\begin{aligned}
& \Omega_{\xi}=\frac{-12 B\left(\xi-\frac{R}{2}\right)}{\left(\eta^{2}+\left(\xi-\frac{R}{2}\right)^{2}\right)^{7}}+\frac{6 A\left(\xi-\frac{R}{2}\right)}{\left(\eta^{2}+\left(\xi-\frac{R}{2}\right)^{2}\right)}-\frac{12 B\left(\xi+\frac{R}{2}\right)}{\left(\eta^{2}+\left(\xi+\frac{R}{2}\right)^{2}\right)^{7}}+\frac{6 A\left(\xi+\frac{R}{2}\right)}{\left(\eta^{2}+\left(\xi+\frac{R}{2}\right)^{2}\right)^{4}}+2 \xi m_{c} \omega^{2} \\
& \Omega_{\eta}=\frac{-12 B \eta}{\left(\eta^{2}+\left(\xi-\frac{R}{2}\right)^{2}\right)^{7}}+\frac{6 A \eta}{\left(\eta^{2}+\left(\xi-\frac{R}{2}\right)^{2}\right)^{4}}-\frac{12 B \eta}{\left(\eta^{2}+\left(\xi+\frac{R}{2}\right)^{2}\right)^{7}}+\frac{6 A \eta}{\left(\eta^{2}+\left(\xi+\frac{R}{2}\right)^{2}\right)^{4}}+2 \eta m_{c} \omega^{2}
\end{aligned}
$$

Making use of Eq. (30) and Eq. (31), the values of the second order partial derivatives at the stationary point under consideration are

$$
\begin{gathered}
\Omega_{\xi \xi}^{0}=\frac{6 A}{\left(\eta_{o}^{2}+\left(\xi_{o}-\frac{R}{2}\right)^{2}\right)^{4}}-\frac{12 B}{\left(\eta_{o}^{2}+\left(\xi_{o}-\frac{R}{2}\right)^{2}\right)^{7}}-\frac{12 B}{\left(\eta_{o}^{2}+\left(\xi_{o}+\frac{R}{2}\right)^{2}\right)^{7}}+\frac{6 A}{\left(\eta_{o}^{2}+\left(\xi_{o}+\frac{R}{2}\right)^{2}\right)^{4}}+\frac{168 B\left(\xi_{o}-\frac{R}{2}\right)^{2}}{\left(\eta_{o}^{2}+\left(\xi_{o}-\frac{R}{2}\right)^{2}\right)^{8}}- \\
\frac{48 A\left(\xi_{o}-\frac{R}{2}\right)^{2}}{\left(\eta_{o}^{2}+\left(\xi_{o}-\frac{R}{2}\right)^{2}\right)^{5}}+\frac{168 B\left(\xi_{o}+\frac{R}{2}\right)^{2}}{\left(\eta_{o}^{2}+\left(\xi_{o}+\frac{R}{2}\right)^{2}\right)^{8}}-\frac{48 A\left(\xi_{o}+\frac{R}{2}\right)^{2}}{\left(\eta_{o}^{2}+\left(\xi_{o}+\frac{R}{2}\right)^{2}\right)^{5}}+2 m_{c} \omega^{2} \\
\Omega_{\eta \eta}^{0}=\frac{168 B \eta_{o}^{2}}{\left(\eta_{o}^{2}+\left(\xi_{o}-\frac{R}{2}\right)^{2}\right)^{8}}-\frac{12 B}{\left(\eta_{o}^{2}+\left(\xi_{o}-\frac{R}{2}\right)^{2}\right)^{7}}-\frac{48 A \eta_{o}^{2}}{\left(\eta_{o}^{2}+\left(\xi_{o}-\frac{R}{2}\right)^{2}\right)^{5}}+\frac{6 A}{\left(\eta_{o}^{2}+\left(\xi_{o}-\frac{R}{2}\right)^{2}\right)^{4}}+\frac{168 B \eta_{o}^{2}}{\left(\eta_{o}^{2}+\left(\xi_{o}+\frac{R}{2}\right)^{2}\right)^{8}}- \\
\frac{12 B}{\left(\eta_{o}^{2}+\left(\xi_{o}+\frac{R}{2}\right)^{2}\right)^{7}}-\frac{48 A \eta_{o}^{2}}{\left(\eta_{o}^{2}+\left(\xi_{o}+\frac{R}{2}\right)^{2}\right)^{5}}+\frac{6 A}{\left(\eta_{o}^{2}+\left(\xi_{o}+\frac{R}{2}\right)^{2}\right)^{4}}+2 m_{c} \omega^{2}
\end{gathered}
$$

and

$$
\Omega_{\xi \eta}^{0}=\frac{168 B \eta_{o}\left(\xi_{o}-\frac{R}{2}\right)}{\left(\eta_{o}^{2}+\left(\xi_{o}-\frac{R}{2}\right)^{2}\right)^{8}}-\frac{48 A \eta_{o}\left(\xi_{o}-\frac{R}{2}\right)}{\left(\eta_{o}^{2}+\left(\xi_{o}-\frac{R}{2}\right)^{2}\right)^{5}}+\frac{168 B \eta_{o}\left(\xi_{o}+\frac{R}{2}\right)}{\left(\eta_{o}^{2}+\left(\xi_{o}+\frac{R}{2}\right)^{2}\right)^{8}}-\frac{48 A \eta_{o}\left(\xi_{o}+\frac{R}{2}\right)}{\left(\eta_{o}^{2}+\left(\xi_{o}+\frac{R}{2}\right)^{2}\right)^{5}}
$$




$$
\Omega_{\eta \xi}^{0}=\frac{168 B \eta_{o}\left(\xi_{o}-\frac{R}{2}\right)}{\left(\eta_{o}^{2}+\left(\xi_{o}-\frac{R}{2}\right)^{2}\right)^{8}}-\frac{48 A \eta_{o}\left(\xi_{o}-\frac{R}{2}\right)}{\left(\eta_{o}^{2}+\left(\xi_{o}-\frac{R}{2}\right)^{2}\right)^{5}}+\frac{168 B \eta_{o}\left(\xi_{o}+\frac{R}{2}\right)}{\left(\eta_{o}^{2}+\left(\xi_{o}+\frac{R}{2}\right)^{2}\right)^{8}}-\frac{48 A \eta_{o}\left(\xi_{o}+\frac{R}{2}\right)}{\left(\eta_{o}^{2}+\left(\xi_{o}+\frac{R}{2}\right)^{2}\right)^{5}}
$$

Eq. (34) and Eq. (35) are equal. That is $\Omega_{\xi \eta}^{0}=\Omega_{\eta \xi}^{0}$, therefore Eq. (30) becomes

$$
\lambda^{4}-\left[\frac{1}{2 m_{c}}\left(\Omega_{\xi \xi}^{0}+\Omega_{\eta \eta}^{0}-8 \omega^{2} m_{c}\right)\right] \lambda^{2}+\left[\frac{1}{4 m_{c}^{2}}\left(\Omega_{\xi \xi}^{0} \Omega_{\eta \eta}^{0}-\left(\Omega_{\xi \eta}^{0}\right)^{2}\right)\right]=0
$$

Rewriting Eq. (36) we have

$$
\lambda^{4}-\left[\frac{1}{2 m_{c}}\left(G+F-8 \omega^{2} m_{c}\right)\right] \lambda^{2}+\left[\frac{1}{4 m_{c}^{2}}\left(G F-H^{2}\right)\right]=0
$$

where;

$$
\begin{aligned}
& G=\frac{6 A}{\left(\eta_{o}^{2}+\left(\xi_{o}-\frac{R}{2}\right)^{2}\right)^{4}}-\frac{12 B}{\left(\eta_{o}^{2}+\left(\xi_{o}-\frac{R}{2}\right)^{2}\right)^{7}}-\frac{12 B}{\left(\eta_{o}^{2}+\left(\xi_{o}+\frac{R}{2}\right)^{2}\right)^{7}}+\frac{6 A}{\left(\eta_{o}^{2}+\left(\xi_{o}+\frac{R}{2}\right)^{2}\right)^{4}}+\frac{168 B\left(\xi_{o}-\frac{R}{2}\right)^{2}}{\left(\eta_{o}^{2}+\left(\xi_{o}-\frac{R}{2}\right)^{2}\right)^{8}}-\frac{48 A\left(\xi_{o}-\frac{R}{2}\right)^{2}}{\left(\eta_{o}^{2}+\left(\xi_{o}-\frac{R}{2}\right)^{2}\right)^{5}}+ \\
& \frac{168 B\left(\xi_{o}+\frac{R}{2}\right)^{2}}{\left(\eta_{o}^{2}+\left(\xi_{o}+\frac{R}{2}\right)^{2}\right)^{8}}-\frac{48 A\left(\xi_{o}+\frac{R}{2}\right)^{2}}{\left(\eta_{o}^{2}+\left(\xi_{o}+\frac{R}{2}\right)^{2}\right)^{5}}+2 m_{c} \omega^{2} \\
& F=\frac{168 B \eta_{o}^{2}}{\left(\eta_{o}^{2}+\left(\xi_{o}-\frac{R}{2}\right)^{2}\right.}-\frac{12 B}{\left(\eta_{o}^{2}+\left(\xi_{o}-\frac{R}{2}\right)^{2}\right)^{7}}-\frac{48 A \eta_{o}^{2}}{\left(\eta_{o}^{2}+\left(\xi_{o}-\frac{R}{2}\right)^{2}\right)^{5}}+\frac{6 A}{\left(\eta_{o}^{2}+\left(\xi_{o}-\frac{R}{2}\right)^{2}\right)^{4}}+\frac{168 B \eta_{o}^{2}}{\left(\eta_{o}^{2}+\left(\xi_{o}+\frac{R}{2}\right)^{2}\right)^{8}}-\frac{12 B}{\left(\eta_{o}^{2}+\left(\xi_{o}+\frac{R}{2}\right)^{2}\right)^{7}}- \\
& \frac{48 A \eta_{o}^{2}}{\left(\eta_{o}^{2}+\left(\xi_{o}+\frac{R}{2}\right)^{2}\right)^{5}}+\frac{6 A}{\left(\eta_{o}^{2}+\left(\xi_{o}+\frac{R}{2}\right)^{2}\right)^{4}}+2 m_{C} \omega^{2} \text { and } \\
& H=\frac{168 B \eta_{o}\left(\xi_{o}-\frac{R}{2}\right)}{\left(\eta_{o}^{2}+\left(\xi_{o}-\frac{R}{2}\right)^{2}\right)^{8}}-\frac{48 A \eta_{o}\left(\xi_{o}-\frac{R}{2}\right)}{\left(\eta_{o}^{2}+\left(\xi_{o}-\frac{R}{2}\right)^{2}\right)^{5}}+\frac{168 B \eta_{o}\left(\xi_{o}+\frac{R}{2}\right)}{\left(\eta_{o}^{2}+\left(\xi_{o}+\frac{R}{2}\right)^{2}\right)^{8}}-\frac{48 A \eta_{o}\left(\xi_{o}+\frac{R}{2}\right)}{\left(\eta_{o}^{2}+\left(\xi_{o}+\frac{R}{2}\right)^{2}\right)^{5}}
\end{aligned}
$$

\section{Numerical Applications}

Considering Eq. (21), Eq. (22) and Eq. (37), the locations and stability of the stationary points of the carbon atom in the vicinity of two $C_{60}$ fullerenes are computed numerically using the software package MATHEMATICA. The positions and stability of the aforementioned problem are shown in Table 2, Fig. 2 and Fig. 3 and Table 3 respectively. The total pairwise molecular interactions between two $C_{60}$ fullerenes, which is an approximation of a continuous approach is termed the total molecular energy evidenced in Eq. (2). As it is evidenced in Table 3 below, for each set of values, there is at least one complex root with the positive real part. Hence in the Lyapunov sense, they are unstable. This agrees with the works of [9-15].

Table 3: Stability of collinear equilibrium points of the carbon atom for $A=17.4, B=29 \times 10^{3}$, $R=10.5, m_{c}=1.993 \times 10^{-26}, \omega=6 \times 10^{11}$

\begin{tabular}{cccc}
\hline \hline $\boldsymbol{\xi}$ & $\pm \boldsymbol{\eta}$ & $\pm \boldsymbol{\lambda}_{\mathbf{1 , 2}}$ & $\pm \lambda_{3,4}$ \\
\hline $\mathbf{0}$ & 0 & $-2.26811 \times 10^{11} \pm 6.55746 \times 10^{11} i$ & $2.26811 \times 10^{11} \pm 6.55746 \times 10^{11} i$ \\
$-\mathbf{8 . 3 2 2 8 5}$ & 0 & $-3.77537 \times 10^{12} \pm 1.13828 \times 10^{12} i$ & $3.77537 \times 10^{12} \pm 1.13828 \times 10^{12} i$ \\
$\mathbf{8 . 3 2 2 8 5}$ & 0 & $-3.77537 \times 10^{12} \pm 1.13828 \times 10^{12} i$ & $3.77537 \times 10^{12} \pm 1.13828 \times 10^{12} i$ \\
\hline \hline
\end{tabular}

\section{Discussion and Conclusion}

We have examined the classical restricted three-body problem of a carbon atom in the vicinity of two $C_{60}$ fullerenes at the nanoscale. The total molecular energy between the two fullerenes are determined analytically by approximating the pairwise potential energies between the carbon atoms on the fullerenes by a continuous approach. Using software MATHEMATICA, we have computed the positions and stability of the stationary points of a carbon atom at the nanosacle. The positions and stability of the aforementioned problem are shown in Table 2, Fig. 2, Fig. 3 and Table 3 respectively. 
The positions of the stationary points as indicated in Table 2, Fig. 2 and Fig. 3 show that they are collinear points lying on the $\xi$ - axis which are symmetric about the $\eta-$ axis, since the carbon are evenly distributed about the nucleus.

The total pairwise molecular interactions between two $C_{60}$ fullerenes, which is an approximation of a continuous approach is termed the total molecular energy as shown in Eq. (2). Upon using Eq. (14) and Eq. (15), we can predict the collective angular velocity $\omega$, since the centripetal forces between the three fullerenes are provided by the attractive Van der Waals forces between only molecules. Since only attractive Van der Waals forces contribute to the orbiting behavior, no orbiting phenomenon can be observed for $r<10{ }_{A}^{\circ}$, where the Van der Waals forces becomes repulsive. Assuming zero friction, this model implies that the $C_{60}$ fullerenes oscillates at constant velocity and the instantaneous forces at the extremities serve only to change direction. Our model predicts the oscillating frequencing of the $C_{60}$ molecule to be in the gigahertz range, which is in agreement with molecular dynamical simulations. We observed that the effect of gravity seems to be negligible as we have recorded minimum energy position of both the offset atom and $C_{60}$ fullerenes. The centrifugal forces affect the position of the $C_{60}$ fullerenes, shifting their minimum energy position away from the center of the nanotorus ring as shown in Fig. 2 and Fig. 3. For both the energy and the location of both the offset atom and the $C_{60}$ fullerenes, our model agrees with corresponding results from Eq. [3]. Although the $C_{60}$ - nanotorus orbital is speculative in nature and also presents exciting possibilities, there are still many practical challenges that would need to be overcome before the $C_{60}-$ nanotorus orbital might be realized. However, the present theoretical study is a necessary precursor to any of such developments. As it is evidenced from Table 3, for each set of values at least one root is a complex root with positive real part, hence in the Lyapunov sense, the stationary points are unstable.

\section{Conflict of Interest}

On behalf of all authors, the corresponding author states that there is no conflict of interest.

\section{Acknowledgement}

We are indelibly indebted to Dr Aishetu Umar and Mr. Solomon Okpanachi Omale. Their contributions in the choice of words, numerical works, presentation of tables and figures have enriched this manuscript. We owe you a great deal of gratitude.

\section{References}

[1] D. E. H. Jones, Hollow molecules. New science 32 (1966) 245.

[2] H. W. Kroto, J.R. Heath, S.C. O’Brien, R.F. Curl, R. E. Smalley, $C_{60}$ Buckminster fullerene. Nature 318 (1985) 162-163.

[3] Q. Zhen, J. Z. Liu, Q. Jiang, Excess Van der Waals interaction energy of a multi-walled carbon nanotude with an extruded core and the induced core oscillation. Phys. Rev. B. 65 (2002) 245.

[4] P. Liu, Y.W. Zhang, C. Lu, Oscillatory behavior of $C_{60}$-nanotube oscillators. A molecular dynamics study. Journal of applied physics 97 (2005).

[5] B. J. Cox, N. Thamwattana, J. M. Hill, Mechanics of atoms and fullerenes in single-walled carbon nanotubes. Acceptance and suction energies. Proc. Roy. Soc. London A. 463 (2007) 461.

[6] Y. Chan, G. M. Cox, J. M. Hill, A carbon orbiting around the outside of a carbon nanotube. In proceedings of International Conference on Nanoscience and Nanotechnology ICONN 2008 (2008) 152-155.

[7] Y. Chan, N. Thamwattana, J. H. Hill, Restricted three-body problems at the nanoscale. Conditional mathematics-material science 62 (2009) 25.

[8] J.E.L. Jones, The determination of molecular fields. From the variation of the viscosity of a gas with temperature. Proc. Roy. Soc., 106A (1924) 441. 
[9] C.N. Douskos, V.V. Markellos, Out-of-Plane equilibrium points in the restricted three-body problem with oblateness. Astronomy and Astrophysics, 466 (2006) 357-362.

[10] M.K. Das, P. Narang, S. Mahajan, M. Yuasa, On out of plane equilibrium points in PhotoGravitational restricted three-body problem Astrophys. Astr. 30 (2009) 177-185.

[11] J. Singh, A. Umar, On the stability of triangular equilibrium points in the elliptic R3BP under radiating and oblate primaries. Astrophys. Space Sci. 341 (2012) 349-358.

[12] J. Singh, A. Umar, On out of plane equilibrium points in the Elliptic restricted three-body problem with radiation and oblate primaries. Astrophys. Space Sci. 344 (2013) 13-19.

[13] J. Singh, T.O. Amuda, Out-of-Plane equilibrium points in the photogravitational circular restricted three-body problem with oblateness and P-R Drag. Astronomy and Astrophysics 36 (2015) 291-305.

[14] J. Singh, K. R. Tyokyaa, Stability of triangular points in the elliptic restricted three-body problem with oblateness up to zonal harmonic $\mathrm{J}_{4}$ of both primaries. Eur. Phys. J. Plus, 131 (2016) 365.

[15] J. Singh, K. R. Tyokyaa, Stability of collinear points in the elliptic restricted three-body problem with oblateness up to zonal harmonic $\mathrm{J}_{4}$ of both primaries. Eur. Phys. J. Plus, 132 (2017) 330. 